\title{
Automatic and Online Detection of Rotor Fault State
}

\author{
Ali Ouanas ${ }^{* *}$, Ammar Medoued*, Salim Haddad**, Mourad Mordjaoui*, Djamel \\ Sayad*
}

*Department of Electrical Engineering, University 20 Aout 1955-Skikda. B.P.26 Route El-Hadaiek, Skikda 21000 Algeria.

**Department of Mechanical Engineering, University 20 Aout 1955-Skikda. B.P.26 Route El-Hadaiek, Skikda 21000 Algeria.

\begin{abstract}
In this work, we propose a new and simple method to insure an online and automatic detection of faults that affect induction motor rotors. Induction motors now occupy an important place in the industrial environment and cover an extremely wide range of applications. They require a system installation that monitors the motor state to suit the operating conditions for a given application. The proposed method is based on the consideration of the spectrum of the single-phase stator current envelope as input of the detection algorithm. The characteristics related to the broken bar fault in the frequency domain extracted from the Hilbert Transform is used to estimate the fault severity for different load levels through classification tools. The frequency analysis of the envelope gives the frequency component and the associated amplitude which define the existence of the fault. The clustering of the indicator is chosen in a two-dimensional space by the fuzzy c mean clustering to find the center of each class. The distance criterion, the K-Nearest Neighbor (KNN) algorithm and the neural networks are used to determine the fault type. This method is validated on a $5.5-\mathrm{kW}$ induction motor test bench.
\end{abstract}

Keywords: : broken bar fault, Fuzzy c mean clustering, Hilbert Transform, neural network, KNN, envelope.

Article History: Received July 16 th 2017 ; Received: October 5th 2017; Accepted: January $6^{\text {th }}$ 2018; Available online

How to Cite This Article: Ouanas, A., Medoued, A., Haddad, S., Mordjaoui, M., and Sayad, D. (2017) Automatic and online Detection of Rotor Fault State. International Journal of Renewable Energy Development, 7(1), 43-52.

http://dx.doi.org/10.14710/ijred.7.1.43-52

\section{Introduction}

Due to their great benefits, induction motors are widely used in industry. The industrial processes require good reliability and safety of machines operation. However, the unexpected failure of the machines leads to losses in the production process, in addition to the very high cost of maintenance and long stopping times that may cause. Usually, regular maintenance and planned maintenance schedules are carried out in the aim of detecting problems in the machine before resulting in catastrophic failures (Tavner, 2008). Therefore, a monitoring system is quite necessary to improve the availability and to increase the expected lifetime in various domains like electricity generation (Rahman et al., 2017; Toke, 2015; Sreedharan et al., 2011; Munshi and Sampath, 2016; McLaughlin and Pearce, 2013; Benakcha et al. 2017). In fact, the monitoring of the proper functioning of the machine is becoming quite useful and increasingly crucial to eliminate any negative impact.

In recent years, researchers have studied several varieties of machine faults, such as eccentricity faults, bearing faults, winding faults and broken bars faults. This latter represents about $7 \%$ of the totality of the induction motor breakdowns (Bonnett and Yung, 2008).
When one bar of the squirrel cage rotor is broken, it may not cause an immediate failure in the induction motor, but rather an increase of vibrations in the machine, oscillations in the stator current, changes in temperature, etc. In case the fault persists and the induction motor continues to operate, the rotor will probably have more broken bars and symptoms of faults multiply. This makes the detection of the first broken bar a crucial priority to avoid any kind of serious damage of the induction motor.

Many diagnosis methods have experienced several perfections to be able to detect the faults that prevent the normal operation of the machine. Recently the monitoring of the imperfections behavior of the machines is becoming a major interest for the researchers. A variety of solutions have been proposed in order to ensure the diagnosis accuracy and reliability. among these monitoring technique, the motor current signature analysis (MCSA) method which has experienced a huge success of stator and rotor faults detection (Benbouzid, 2000; Lebaroud and Medoued, 2013). The signal processing techniques such as wavelets (Ordaz-Moreno et al., 2008) and wavelet packet decomposition in which we can pull the signatures of faults from the stator current (Ye, Wu, \& Zargari, 2000)

\footnotetext{
* Corresponding author: amedoud75@yahoo.fr
} 
In fact, the use of monitoring techniques and the conventional detection has moved to the exploitation phase using artificial intelligence methods, such as Artificial Neural Networks (Boukra, Lebaroud, \& Clerc Guy, 2013; Medoued et al., 2014), Fuzzy Logic (Laala et al., 2014) and Neuro-Fuzzy (Ballal et al., 2007). Neural Network (NN) techniques are used for classification and identification after the detection of the broken bars, the windings and the eccentricity faults using the wavelet technique (Sadeghian, Ye, \& Wu, 2009). The Neuron Network (NN) techniques can perform fault detection without need of the complex and rigorous mathematical model. They are quite flexible in solving some nonlinear problems.

The current signal contains a potential of important information on the existence of the different faults (short circuit, broken bar, bearing fault, etc.). The current signals the magnitude is easy to measure using a current transformer while the system is operating. This advantage has allowed the MCSA method to ensure an online fault diagnosis such as the broken bar fault. The analysis of the current spectrum is used to evaluate the state of the rotor. The harmonics at the frequency $(1 \pm 2 \mathrm{ks}) f_{s}\left(f_{s}\right.$ is the supply frequency, $s$ is the slip and $\mathrm{k}=1,2,3)$ indicate the presence of fault. The motor current signature analysis MCSA is largely employed because of its simplicity and its online detection character. On the other hand, this technique has certain practical limits in industrial environment for several reasons.

The MCSA method is based on the precision of the FFT. The limitation of the data measurement produces the spectral leakage (Culbert, \& Rhodos, 2005). The magnitudes of left and right sideband frequency components are very small compared to the magnitudes of the fundamental frequency. The energy of this latter propagates and may easily hide the

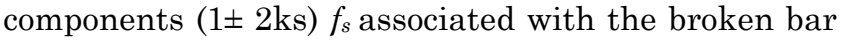
fault which influences the detection performance.

The spectral leakage exhibits a negative impact on the frequency resolution when the motor operates with a weak slip. The left and right sideband components are very close to the fundamental frequency. In this case, the fault detection becomes quite difficult. Consequently, to overcome this problem, the frequency high resolution is used to insure a precise separation (Kia et al., 2007). On the other hand, the MCSA method has certain disadvantages which face the industrial applications (Puche-Panadero, \& al., 2009).

The difficulties of the MCSA method, and especially the broken bar fault detection with weak slip are solved by an alternative MCSA based on Hilbert Transform. It relies on the spectrum of only one current phase (Kia, Henao, \& Capolino, 2009). The spectral analysis of the analytical signal magnitude presents significant advantages in comparison with the traditional MCSA. This method is very suitable for online and offline detection in practical applications.

The frequency components related to the broken bar fault appear on the spectrum of the instantaneous amplitude extracted from Hilbert Transform (Kia,
Henao, \& Capolino, 2009). Comparative studies in (Puche-Panadero, \& al., 2009; Jimenez, \& al., 2007; Wang, \& al., 2012) showed that the Hilbert envelope spectrum provides harmonics in the lower frequency band which characterizes better the broken bar fault compared with the traditional MCSA. Detection with weak slip constitutes a real challenge for the traditional MCSA.

The time domain method based on the application of the discrete wavelet transform DWT on the stator current space vector magnitude and the instantaneous magnitude of the stator current signal is proposed in (Kia, Henao, \& Capolino, 2009), the obtained results show the possibility to detect the broken bar fault without the slip estimation by the calculation of the energy associated with the low frequency bandwidth. The amplitude and the frequency (characteristic peak) of the Hilbert envelope spectrum present a good indicator for the classification based on the Support vector machines SVM. In (Matić, \& al., 2012) several kernel functions are used such as linear, quadratic and gaussian to evaluate the detection performance with weak slips. The broken bar fault produces fluctuations within the instantaneous amplitude. The term $2 f_{s}$ is proportional with the slip (Ghorbanian, \& Faiz, 2015). The amplitude and the frequency of the harmonic $2 f_{s}$ of the envelope spectrum are very sensitive to the broken bar fault. In (Laala, \& al., 2014), the amplitude of the harmonic $2 f_{s}$ and the DC component are used to detect the number of the broken bars using Fuzzy logic.

The induction motor can be fed in two ways: networks and frequency converter. The network feeding provides a pure sinusoidal signal to the motor, whereas, the frequency converter signal suffers from various harmonics. The herein suggested method consists of applying the Hilbert Transform on only one current phase of a motor. The motor is supplied by a converter in the training phase to check the sensitivity of the envelope spectrum harmonics which presents a very interesting discriminatory criterion to build an expert system which can detect the broken bar fault. The two-dimensional plane (frequency, amplitude) is used to determine the centers of each class using fuzzy c mean cluster (Aydin, Karakose, \& Akin, 2007). The classification task is carried out by the Euclidean distance criterion and KNN. The Multilayer Perceptron (MLP) Neural Networks is used in this work to automatically estimate the number of the broken bars and compare the results with the proposed method.

\section{Hilbert transform}

The Hilbert transform (HT) is a signal demodulation method, used in the faults diagnostic field such as bearing faults (Medoued and al., 2016). The extraction of the envelope allows us to identify indicators which characterize the appearance of faults (Matić and al., 2012; Laala et al., 2014)., The Hilbert transform $\mathrm{H}\left[c_{i}(t)\right]$ is considered as a signal processing tool used in 
different scientific fields ( King, 2009). The HT is defined by:

$$
\mathrm{H}\left[c_{i}(t)=\frac{1}{\pi} \int_{-\infty}^{\infty} \frac{c_{i}(\tau)}{(t-\tau)} d \tau\right.
$$

The analytical signal $z_{i}(t)$ is defined by:

$$
z_{i}(t)=c_{i}(t)+j \mathrm{H}\left[c_{i}(t)\right]
$$

or in an exponential representation:

$$
z_{i}(t)=a_{i}(t) \exp \left(j \omega_{i}(t)\right)
$$

The instantaneous amplitude is given by:

$$
a_{i}(t)=\sqrt{c_{i}^{2}(t)+H^{2}\left[c_{i}(t)\right]}
$$

\section{Experimental results}

The Bench consists of a $5.5 \mathrm{~kW}$ three-phase squirrel cage induction motor Figure 1. The motor is Leroy Somer LS 132s, IP 55, Class F, standardized $\mathrm{T}^{\circ} \mathrm{C}=$ $40^{\circ} \mathrm{C}$. The nominal voltage between phases: $400 \mathrm{~V}$, the frequency of power supply $50 \mathrm{~Hz}$, nominal speed 1440 $\mathrm{rpm}$, the rotor slots number $\mathrm{NR}=28$. The stator slots number $\mathrm{ns}=48$. The stator windings are coupled in star. The motor is loaded by a Powder Brake. Its maximum torque is $100 \mathrm{~nm}$ reached at the nominal speed.

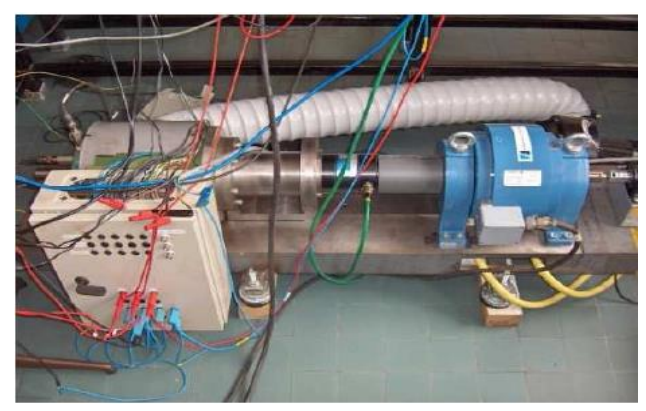

Fig.1. Test bench

The rotor broken bar fault is created by drilling holes (Figure 2).

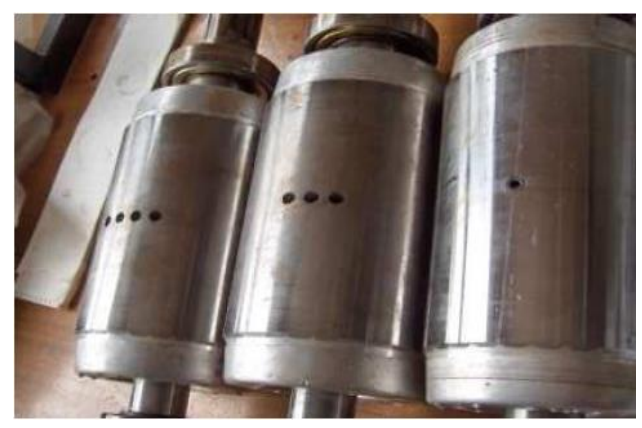

Fig. 2. Creation of broken bars.

\subsection{The extraction of the envelope}

The Hilbert Transform gives the instantaneous amplitude of the current signal powered by a frequency inverter on a 3-broken bar faulted machine Figure 3.

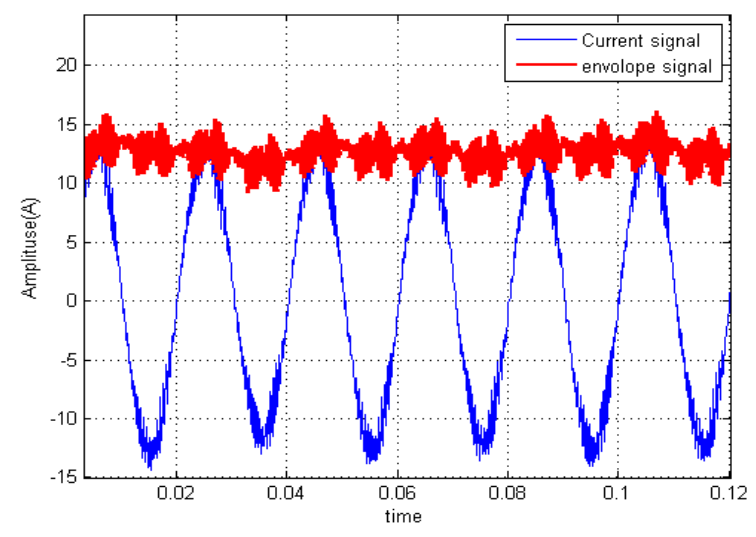

Fig.3. Instantaneous amplitude of three broken bars.

The obtained envelope is processed using FFT. As shown in Figure 4, the instantaneous amplitude spectrum (with 25\%, 50\% and 75\% load level) shows that the important components are localized in the lower frequency band. The spectral analysis of the amplitude shows that the harmonic $\left(2 f_{s}\right)$ are detached from the rest of the extended frequency spectrum. This harmonic, experimentally obtained, reveals the existence of the broken bar fault.
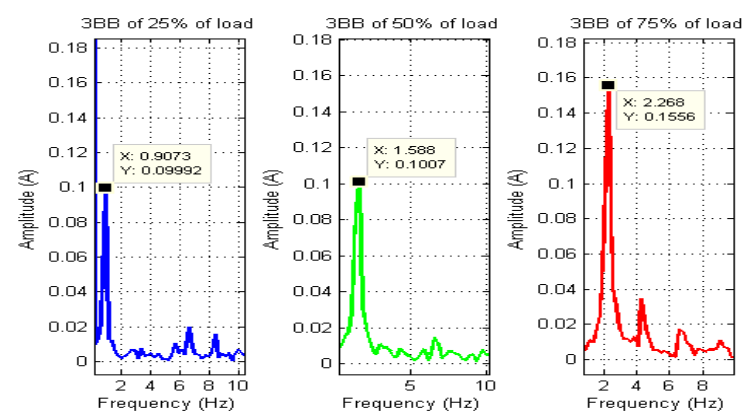

Fig.4. Envelope spectrum for (25\%, 50\% and 75\%) load levels of 3-broken bar case.

The harmonic $\left(f_{2 f_{s}}\right)$ position is very sensitive to the variation of the load level. The amplitude is also evaluated with the severity of the fault (number of broken bars). The observation of the two indicators makes it possible to deduce the rotor state and followup the evolution of the frequency component represented in Figure 4 in the spectrum of the broken bar fault as well as the healthy case.

\subsection{Fuzzy c mean clustering}

The frequency observation of the component $\left(2 f_{s}\right)$ and the associated amplitude in the broken bar and healthy spectrums on several load levels $(25 \%, 50$ and $75 \%$ ) allow us to distinguish the rotor state. The data are represented in the two-dimensional space (Freq, Amp) with the help of the Fuzzy c mean (FCM) cluster, a method whose main role is to cluster all the data into healthy and faulty classes. FCM is a data clustering 
Citation: Ouanas, A., Medoued, A., Haddad, S., Mordjaoui, M., and Sayad, D. (2017) Automatic and online Detection of Rotor Fault State. Int. Journal of Renewable Energy Development, 7(1),43-52, doi.org/10.14710/ijred.7.1.43-52

$\mathrm{P}$ a g e 46

technique that allows one point to belong to one or more clusters. The method was developed by Dunn (Dunn, 1978) and improved by Bezdek (Bezdek, 1981). It is frequently used in fault diagnosis domains.

The training data $\left(x_{i}\right)(\Delta)$ of size $\mathrm{N}$ of the component $2 f_{s}$ collected in all load level cases on the plane (amplitude, frequency) is presented in Fig. 5, 6 and 7. " $\mathrm{C}$ " is the cluster number, in our case it is equal to three classes, $\mathrm{C}=\left\{C_{25 \%}, C_{50 \%}\right.$ and $\left.C_{75 \%}\right\}$. For each type of fault, we consider $\left(c_{j}\right)$ the center of each cluster defined by equation (5),

$$
C_{j}=\frac{\sum_{i=1}^{N} u_{i j}^{m} \cdot x_{i}}{\sum_{i=1}^{N} u_{i j}^{m}}
$$

Where $u_{i j}$ is the $x_{i}$ membership degree in the cluster and $u_{i j} \in[0,1]$. The parameter $\mathrm{m} \in[1, \infty]$ controls the weight given to each cluster center.

$$
\mathrm{u}_{\mathrm{ij}}=\frac{1}{\sum_{\mathrm{k}=1}^{\mathrm{c}}\left(\frac{\left\|\mathrm{x}_{\mathrm{i}}-\mathrm{c}_{\mathrm{j}}\right\|}{\left\|\mathrm{x}_{\mathrm{i}}-\mathrm{c}_{\mathrm{k}}\right\|}\right)^{\frac{2}{\mathrm{~m}-1}}}
$$

The optimum value of $c_{j}$ is given by the minimization of the objective function $\mathrm{J}$ given by (7) by solving equations (5) and (6):

$$
J_{m}=\sum_{i=1}^{N} \sum_{j=1}^{C} u_{i j}^{m}\left\|x_{i}-c_{j}\right\|^{2}
$$

The FCM is applied separately to each type of fault in order to have the centers of the different classes.

In Figures 5, 6 and 7, The clustering results were separated in a precise way. They are three classes of cases: healthy, 1-broken bar, 3-broken bars. The centers (x) identify each class. $C_{1}, C_{2}$ and $C_{3}$ are the centers of the healthy class. $C_{4}, C_{5}$ and $C_{6}$ are the centers of 1 -broken bar class. $C_{7}, C_{8}$ and $C_{9}$ are the centers of the 3 -broken bar class. The results confirm that the chosen indicator is very efficient in detecting the broken bar fault.
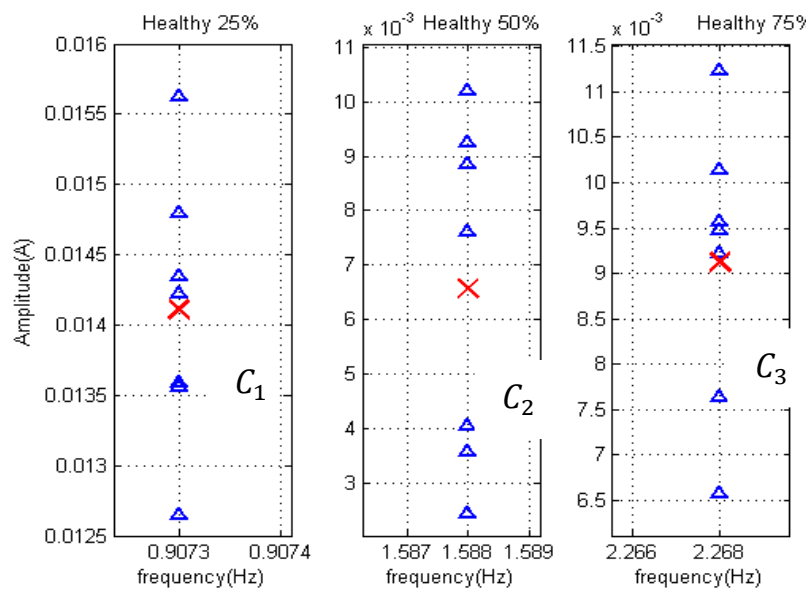

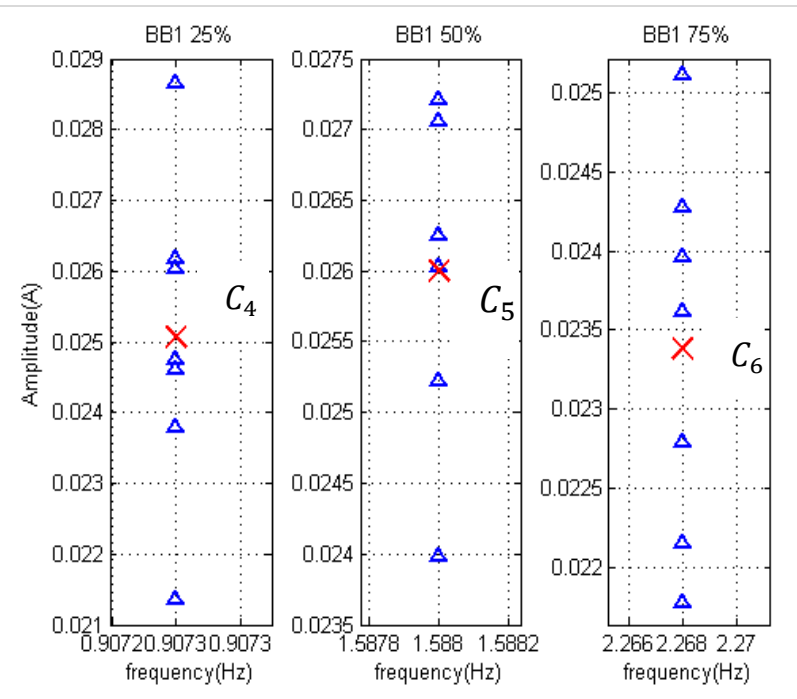

Fig. 6. Centers of 1-broken bar (1BB) classes.

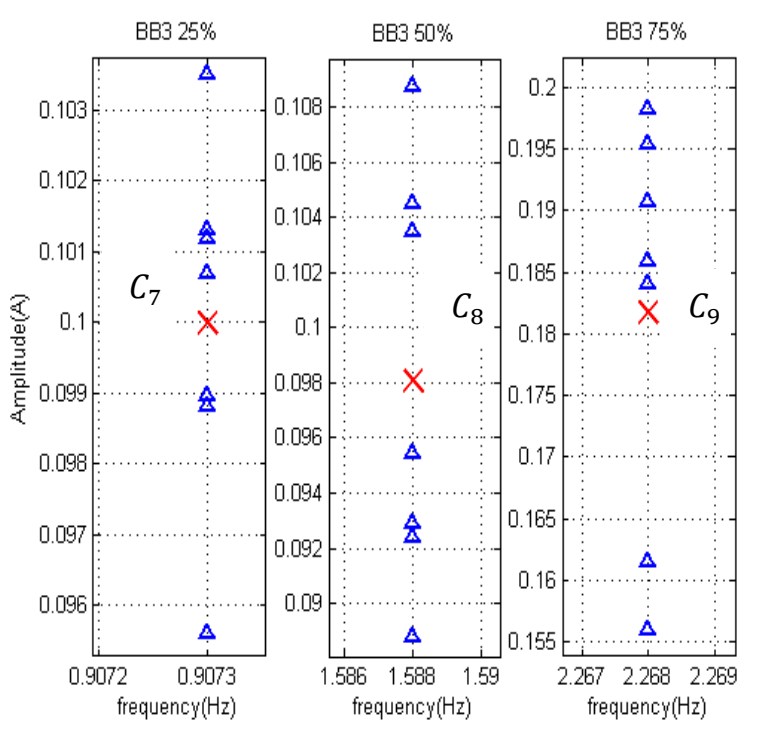

Fig .7. Center of 3-broken bar (3BB) classes.

\subsection{The distance criterion}

The FCM has defined the center $\left(C_{i}\right)$ of the classes. Calculating the Euclidean distance defined in (8) between the test point $(\mathrm{P})$ shown in Figure 8.

$\operatorname{Dist}\left(C_{i}, P\right)=\sqrt{\left(C_{i \text { Freq }}-P_{\text {Freq }}\right)^{2}-\left(C_{i \text { Amp }}-P_{A m p}\right)^{2}}$
$\mathrm{i} \in[1,9] ;$

and the center of each class allows us to obtain the distance confusion matrix.

The minimum value of the Confusion matrix distance allows us to distinguish the class closest to the point (P). As a result, the testing points is classified.

Fig .5. Centers of healthy classes. 
Table 1.

Confusion matrix distance

\begin{tabular}{lcccc}
\hline $\begin{array}{c}\text { Euclidean distance } \\
\text { classification }\end{array}$ & \multicolumn{3}{c}{ Fault severity } \\
\cline { 3 - 5 } & & Healthy & 1BB & 3BB \\
\hline \multirow{2}{*}{ Load } & $75 \%$ & D3 & D6 & D9 \\
level & $50 \%$ & D2 & D5 & D8 \\
\hline
\end{tabular}

Figure 8 shows the distance $(\mathrm{D})$ of the test point $(\mathrm{P})$ and the center of the classes $C_{1}, C_{4}$ and $C_{5}$ which are respectively the center of the healthy class at $25 \%$ load level and the class of $1 \mathrm{BB}$ fault at $25 \%$ load level and the class of $1 \mathrm{BB}$ fault at $50 \%$ load level.

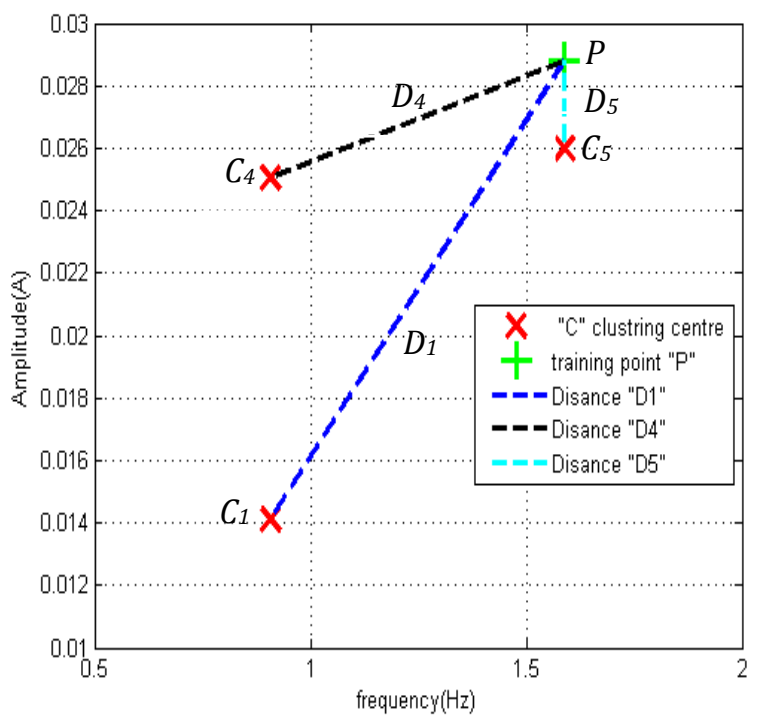

Fig. 8. Distances between the test point $\mathrm{P}$ and the classes centers.

According to Figure 8, the smallest distance is $D_{5}$ and the test point $(\mathrm{P})$ is closer to the center $C_{5}$. It means that $\mathrm{P}$ is a 1 -broken bar fault.

\subsection{K-Nearest Neighbor Algorithm}

The KNN is a simple and very powerful classification algorithm. It is based on the similarity measure (function of the distance) of the samples of a set. Without the training phase the KNN stores all the available cases. It classifies the new cases by calculating the distance matrix. The number $\mathrm{K}$ determines the points closest to the point we want to classify. The KNN algorithm is used in this work to classify the test points defining the classes (healthy and faulty) using FCM.

The main goal is to determine in which class the test point belongs by a majority vote of its neighbors in the healthy or faulty class. Figure 9 represents the test point $\mathrm{P}$ by $(+)$ and the nearest points by (o). It means that $\mathrm{P}$ is a broken bar fault.

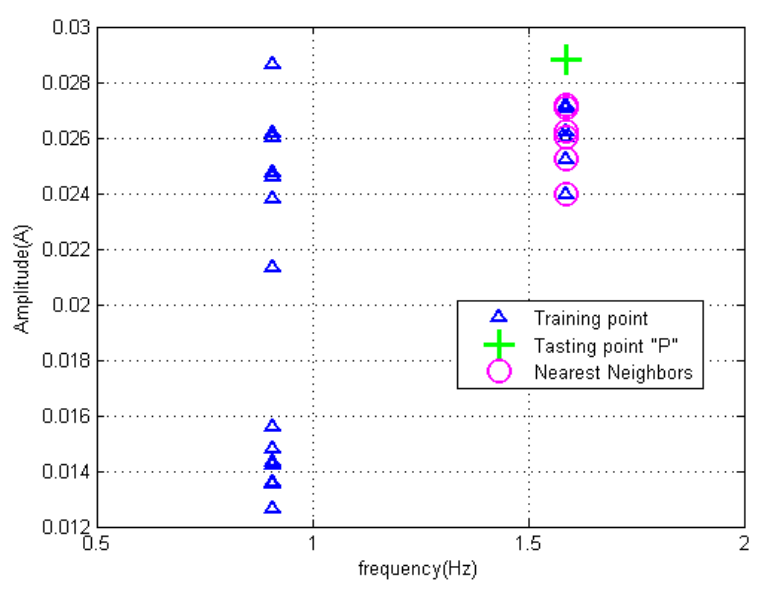

Fig .9. K-Nearest Neighbors of the testing point (P).

\section{Design of the Neuron Network}

In this section, we use Feedforward Multi-Layer Perceptron (MLP) Neural Network and Back Propagation (BP) as a learning algorithm which is widely used in classification problems to automatically detect the broken bar fault. The input of the NN is fixed on the basis of the faulted indicators. In order to obtain an efficient $\mathrm{NN}$ for the detection of broken bar fault .
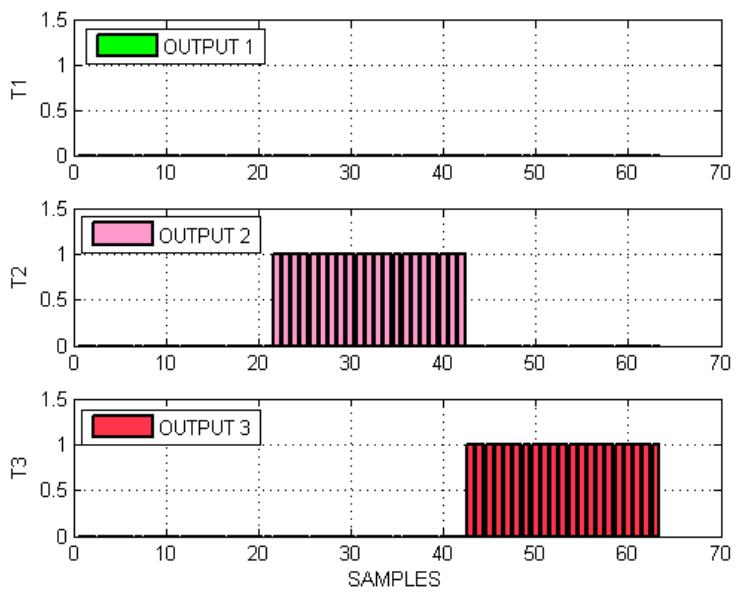

Fig.10. Desired output data set for NN.

The training of the NN is performed by data in Figures 5, 6 and 7, because the designed network should be generalized using the learning data that encompasses the full range, all the conditions as well as all the possible states of operation (healthy and faulty). The NN input [Amp, Freq] of the $2 f_{s}$ component (Figures 57) consists of a row of successive 63 samples. The three operating conditions of the machine: $25 \%, 50 \%$ and $75 \%$ 
Citation: Ouanas, A., Medoued, A., Haddad, S., Mordjaoui, M., and Sayad, D. (2017) Automatic and online Detection of Rotor Fault State. Int. Journal of Renewable Energy Development, 7(1),43-52, doi.org/10.14710/ijred.7.1.43-52

$\mathrm{P}$ a g e $\mid 48$

load level are as follows: Healthy case (21 samples), 1broken bar (21 samples), 3- broken bars (21 samples).

A NN output set of data is necessary to achieve a supervised learning. This set is constructed according to each sample of the input set by the desired output $\mathrm{Ti}$, the obtained target $\mathrm{T}=[\mathrm{T} 1 ; \mathrm{T} 2 ; \mathrm{T} 3]$ is presented in Figure 10, as follows:

- $\mathrm{T}=[0 ; 0 ; 0]$ for $\mathrm{n}=0$; healthy rotor.

- $\mathrm{T}=[0 ; 1 ; 0]$ for $\mathrm{n}=1 ; 1$ - broken bar.

- $\mathrm{T}=[0 ; 0 ; 1]$ for $\mathrm{n}=3 ; 3$ - broken bars.

The used NN includes a single hidden layer. In the aim of finding an optimal structure and the number of neurons of the hidden layer, we start with a few neurons in the hidden layer and make a learning, then we add more neurons until we reach the final number in the hidden layers that gives us a minimum Mean Square Error (MSE).
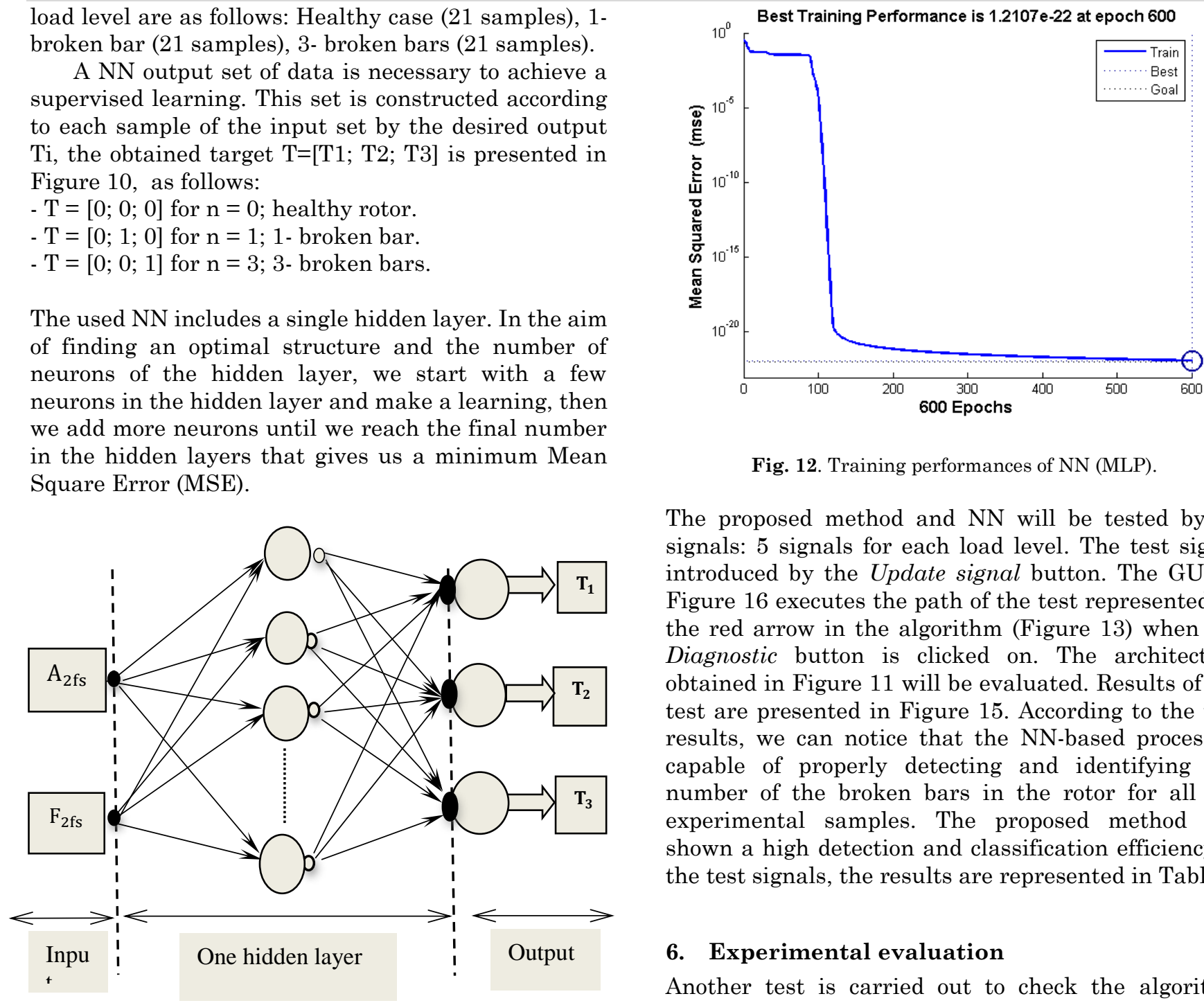

Fig. 12. Training performances of NN (MLP).

The proposed method and NN will be tested by 15 signals: 5 signals for each load level. The test signal introduced by the Update signal button. The GUI of Figure 16 executes the path of the test represented by the red arrow in the algorithm (Figure 13) when the Diagnostic button is clicked on. The architecture obtained in Figure 11 will be evaluated. Results of the test are presented in Figure 15. According to the test results, we can notice that the $\mathrm{NN}$-based process is capable of properly detecting and identifying the number of the broken bars in the rotor for all the experimental samples. The proposed method has shown a high detection and classification efficiency of the test signals, the results are represented in Table 2.

\section{Experimental evaluation}

Another test is carried out to check the algorithm developed in Figure 13 integrated into the GUI of Figure 16. The software has demonstrated a significant detection capability (Table 2). The induction motor of Figure 14 with broken bars is powered by supply network. This procedure allowed us to generate harmonics from the spectrum of the Hilbert envelope as shown in Figure 17. These harmonics are not learned by the GUI to test the detection capability. f "logsig". The NN is capable of producing a desired output with a low error (Figure 12). This shows that the NN has well learned the input data.

\section{GUI of Fault Detection System and Experimental Results}

The detection algorithm shown in Figure 13 includes the training phase of the proposed method and the NN. We introduce a test to see its capacity to detect broken bar fault with experimental data not learnt by the NN and the proposed method. The algorithm of Figure 13 is integrated into a Graphical User Interface (GUI) its main role and to evaluate the test signals acquired from the machine in Figure 14

Table 2.

Results of the proposed method on the frequency inverter.

\begin{tabular}{cccc} 
& Healthy & 1BB & 3BB \\
\hline $\begin{array}{c}\text { Distance } \\
\text { matrices }\end{array}$ & $15 / 15$ & $15 / 15$ & $15 / 15$ \\
$\mathrm{KNN}$ & $15 / 15$ & $15 / 15$ & $15 / 15$ \\
\hline
\end{tabular}




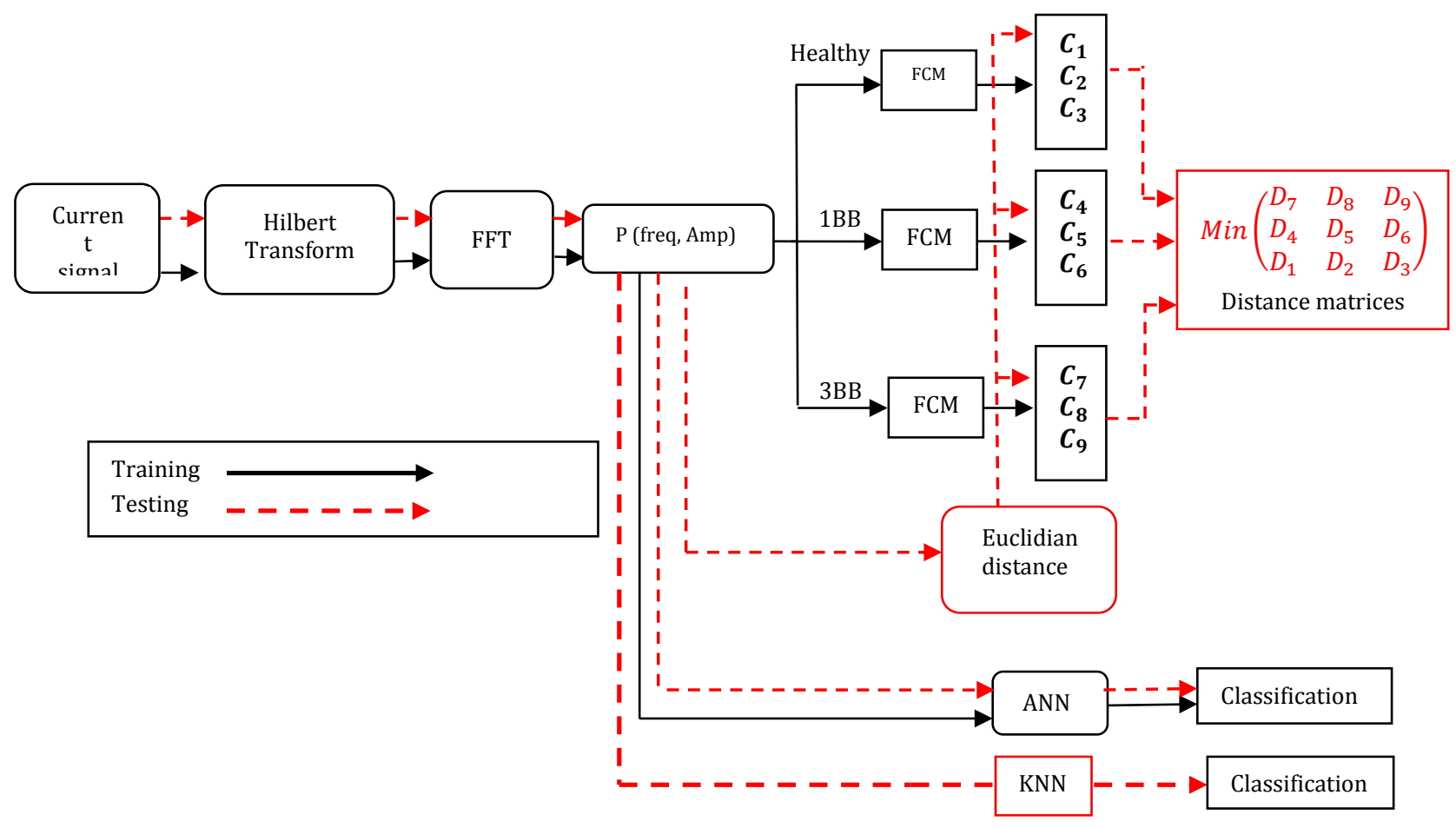

Fig. 13 .The developed software for broken bar fault monitoring

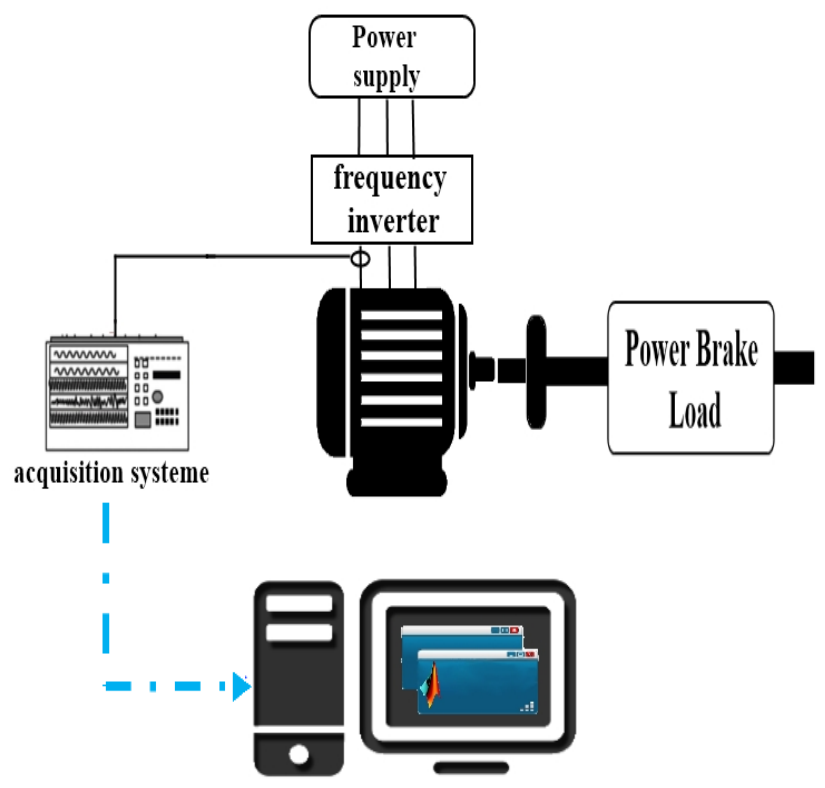

Fig .14. Implementation scheme of the GUI. 
$\mathrm{P}$ a g e $\mid 50$
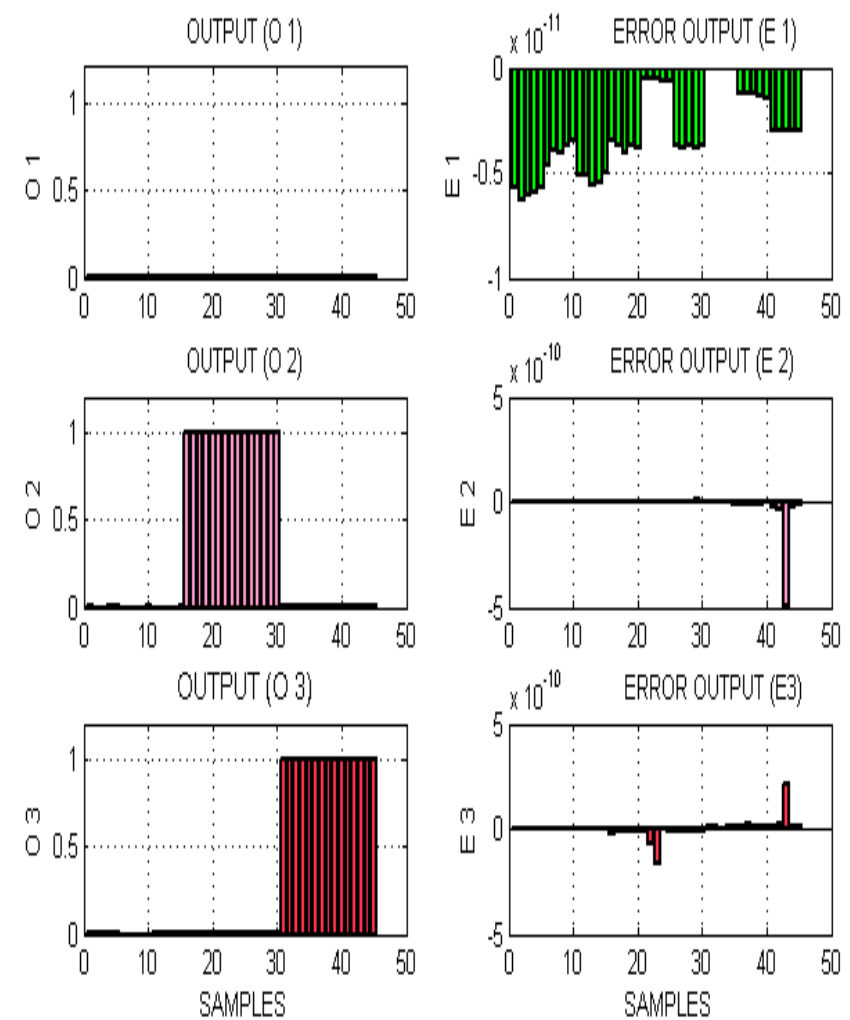

Fig. 15. NN test outputs (left) and error (right).
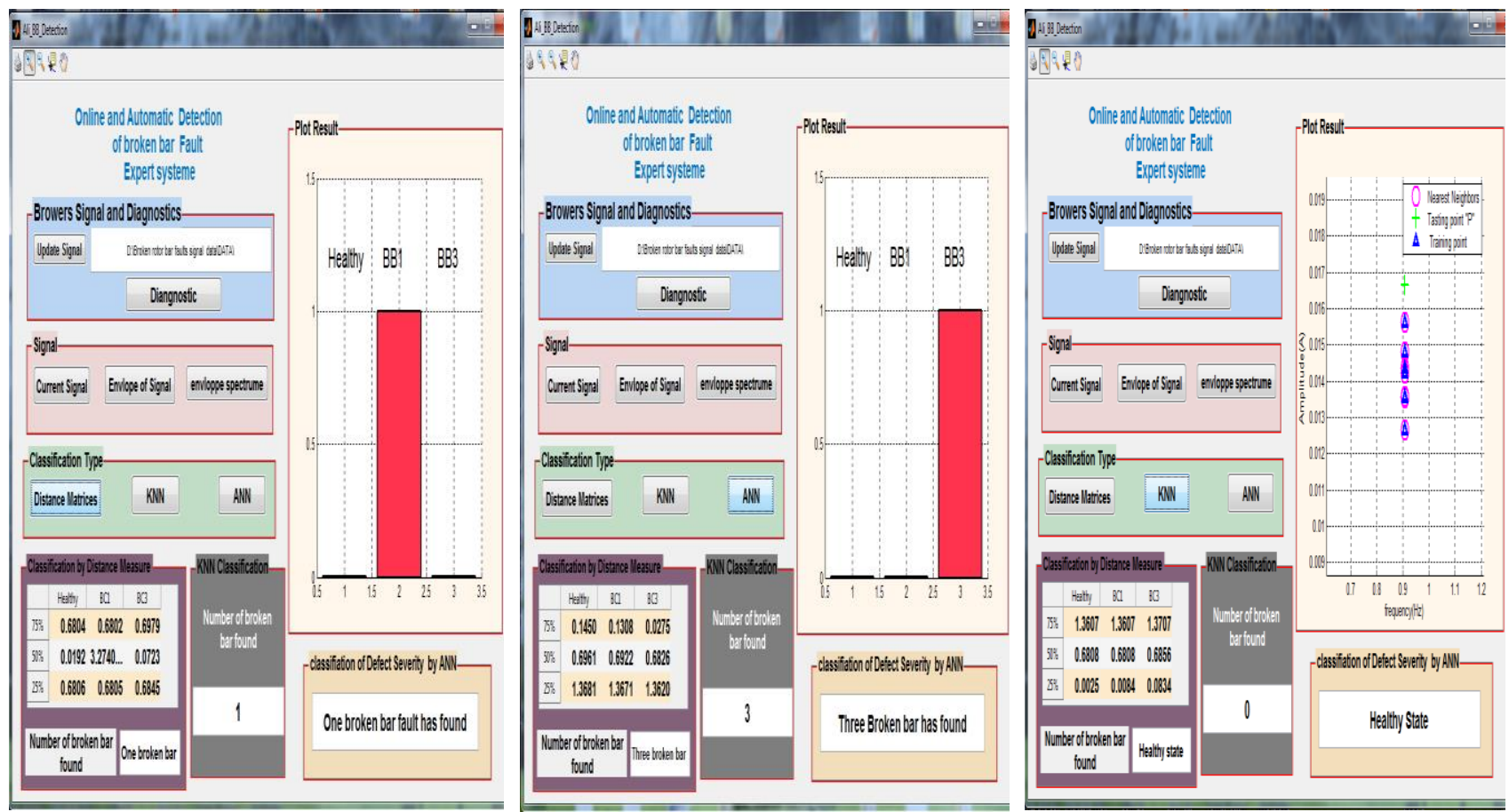

Fig. 16.Developed software for broken bar fault monitoring 


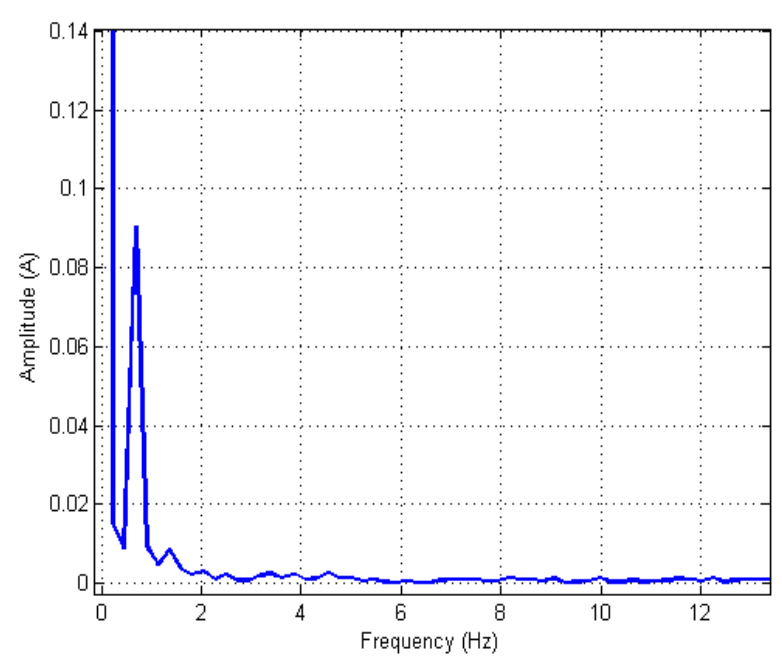

Fig. 17. Spectrum of 3BB of a network-powered motor.

Figure 17 shows the spectrum of the envelope for an induction motor with three broken bars. The obtained spectral peak characterizes the appearance of the fault which has kept the sensitivity to the studied fault.

The classification process of the test points is defined by the red arrow in the algorithm of Figure 16 . We perform a test by calculating the Euclidean distance between the centers of the classes (Figures 5-7) and the spectral peak of Figure 17, the results are summarized in Table 3.

In Table 3 , the minimum distance $(=0.2269)$ is between the test point and the center $\mathrm{C} 7$. This means that the test point is close to the $3 \mathrm{BB}$ fault class at $25 \%$ load level.

Table 3.

Results of Euclidean distance classification.

\begin{tabular}{ccccc}
\multicolumn{2}{c}{$\begin{array}{c}\text { Euclidean } \\
\text { distance } \\
\text { classification }\end{array}$} & \multicolumn{3}{c}{ Fault severity } \\
\hline & & Healthy & $1 \mathrm{BB}$ & $3 \mathrm{BB}$ \\
& $75 \%$ & 1.5904 & 1.5896 & 1.5894 \\
Load & $50 \%$ & 0.9128 & 0.9109 & 0.9076 \\
level & $25 \%$ & 0.2442 & 0.2404 & 0.2269 \\
\hline
\end{tabular}

Another classification method was used in the algorithm of Figure 13 based on the KNN distance criterion. The training phase is carried out by 63 samples (Figures 5-7), each type of fault contains 21 sample. The KNN determines the points closest to the point of the test. The majority vote can define from which class it belongs the points which it is desired to classify.

The KNN calculates the distance between the test point of Figure 17 and all the training data (63 samples) in Figures 5-7. Each class of faults contains 7 samples, the test point of Figure 17 is closer to the three-broken bar fault class at $25 \%$ load level as shown in Figure 18.

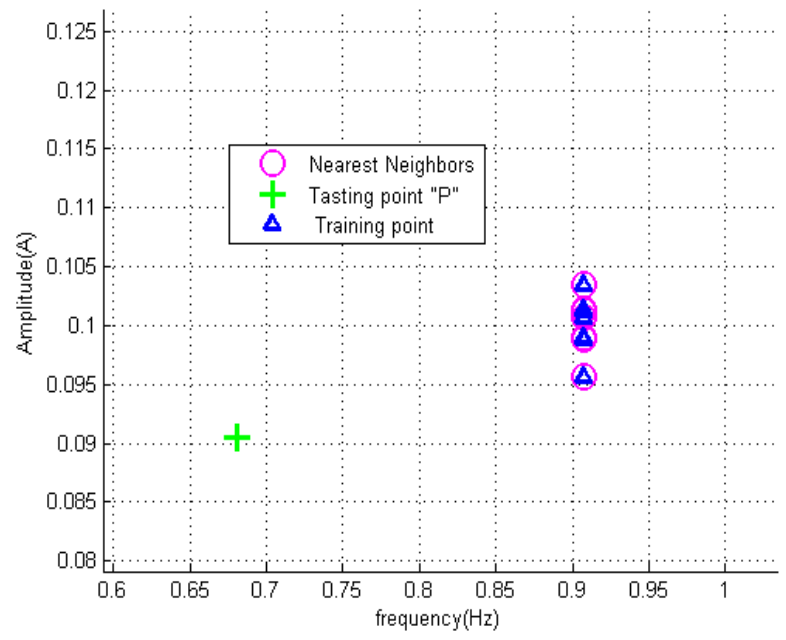

Fig. 18. Nearest class of the testing point (P).

Table 4 summarizes the results accuracy by the proposed methods of healthy cases, $1 \mathrm{BB}$, and $3 \mathrm{BB}$ faults with the load levels $25 \%, 50 \%$ and $75 \%$. The three methods of classification are tested using 45 samples (15 signals for each load level).

The objective of this method is to make discrimination between healthy class and one broken bar class at several load levels. The accuracy obtained by the proposed algorithm is the same of the NN that depends on the chosen indicator which presents a good sensitivity to the fault. On the other hand, the detection with low slip represents the cause of use the MCSA based on Hilbert envelope spectrum. The detection in this case is performed with a small error. The cause of this error could be attributed to the imperfection of the training data set due to a measurement error.

Table 4.

Result of classification accuracy

\begin{tabular}{crcc}
\hline & Healthy & $1 \mathrm{BB}$ & \multicolumn{1}{c}{$3 \mathrm{BB}$} \\
\hline $\begin{array}{c}\text { Distance } \\
\text { matrices }\end{array}$ & $97.77 \%$ & $93.33 \%$ & $100 \%$ \\
$\mathrm{KNN}$ & $97.77 \%$ & $93.33 \%$ & $100 \%$ \\
$\mathrm{NN}$ & $97.77 \%$ & $93.33 \%$ & $100 \%$ \\
\hline
\end{tabular}

\section{Conclusion}

In this work, we present a new approach for automatic and online detection of rotor fault in an induction motor. The approach quantifies automatically the number of the broken bars using the neuron networks, the fuzzy c mean cluster and the distance criterion techniques. The use of indicators from the envelope spectrum gives a good readability of the studied fault by monitoring the amplitude and frequency of the 
Citation: Ouanas, A., Medoued, A., Haddad, S., Mordjaoui, M., and Sayad, D. (2017) Automatic and online Detection of Rotor Fault State. Int. Journal of Renewable Energy Development, 7(1),43-52, doi.org/10.14710/ijred.7.1.43-52

$\mathrm{P}$ a g e $\mid 52$

frequency component $2 f_{s}$. The proposed approach uses the FCM for discrimination by defining the center of each class in the training phase. The distance criterion and the KNN evaluate the test signals. The obtained experimental results show a very high accuracy of detection such as the neural network. The integration of the algorithm in a GUI is very useful for a preventive maintenance and to ensure a reliable diagnosis specifically for this type of fault which threatens the proper functioning of the machine for a given industrial application.

\section{References}

Aydin, I., Karakose, M., \& Akin, E. (2007). A simple and efficient method for fault diagnosis using time series data mining. In Electric Machines, \& Drives Conference, 2007. IEMDC'07. IEEE International (Vol. 1, pp. 596-600). IEEE.

Ballal, M. S., Khan, Z. J., Suryawanshi, H. M., \& Sonolikar, R. L. (2007). Adaptive neural fuzzy inference system for the detection of inter-turn insulation and bearing wear faults in induction motor. IEEE Transactions on Industrial Electronics, 54(1), 250-258.

Benakcha, M., Benalia, L., Ameur, F., \& Tourqui, D. E. (2017). Control of Dual Stator Induction Generator integrated in Wind Energy Conversion System.

Benbouzid, M. E. H. (2000). A review of induction motors signature analysis as a medium for faults detection. IEEE transactions on industrial electronics, 47(5), 984-993.

Bonnett, A. H., \& Yung, C. (2008). Increased efficiency versus increased reliability. IEEE Industry Applications Magazine, 14(1).,

Boukra, T., Lebaroud, A., \& Clerc, G. (2013). Statistical and neural-network approaches for the classification of induction machine faults using the ambiguity plane representation. IEEE Transactions on Industrial Electronics, 60(9), 4034-4042.

Bezdek.J.C (1981). Pattern recognition with fuzzy objective function algoritms. New York: Plenum Press;

Culbert, I., \& Rhodes, W. (2005, September). Using current signature analysis technology to reliably detect cage winding defects in squirrel cage induction motors. In Petroleum and Chemical Industry Conference, 2005. Industry Applications Society 52nd Annual (pp. 95-101). IEEE.

Dunn JC. ( 1978), A fuzzy relative of the ISODATA process and its use in detecting compact well-separated clusters. J Cybernet 3,32-57

Ghorbanian, V., \& Faiz, J. (2015). A survey on time and frequency characteristics of induction motors with broken rotor bars in line-start and inverter-fed modes. Mechanical Systems and Signal Processing, 54, 427-456.

Jimenez, G. A., Munoz, A. O., \& Duarte-Mermoud, M. A. (2007). Fault detection in induction motors using Hilbert and Wavelet transforms. Electrical Engineering, 89(3), 205-220.

Kia, S. H., Henao, H., \& Capolino, G. A. (2007). A high-resolution frequency estimation method for three-phase induction machine fault detection. IEEE Transactions on Industrial Electronics, 54(4), 2305-2314.

Kia, S. H., Henao, H., \& Capolino, G. A. (2009). Diagnosis of broken-bar fault in induction machines using discrete wavelet transform without slip estimation. IEEE Transactions on Industry Applications, 45(4), 1395-1404.

King, F. W. (2009). Hilbert transforms (Vol. 1). New York: Cambridge University Press.

Laala, W., Zouzou, S. E., \& Guedidi, S. (2014). Induction motor broken rotor bars detection using fuzzy logic: experimental research. International Journal of System Assurance Engineering and Management, 5(3), 329-336.

Lebaroud, A., \& Medoued, A. (2013). Online computational tools dedicated to the detection of induction machine faults. International Journal of Electrical Power, \& Energy Systems, 44(1), 752-757.

Matić, D., Kulić, F., Pineda-Sánchez, M., \& Kamenko, I. (2012). Support vector machine classifier for diagnosis in electrical machines: Application to broken bar. Expert Systems with Applications, 39(10), 8681-8689.

McLaughlin, D. V., \& Pearce, J. M. (2013). Progress in indium gallium nitride materials for solar photovoltaic energy conversion. Metallurgical and Materials Transactions A, 44(4), 1947-1954.

Medoued, A., Mordjaoui, M., Soufi, Y., \& Sayad, D. (2016). Induction machine bearing fault diagnosis based on the axial vibration analytic signal. International Journal of Hydrogen Energy, 41(29), 12688-12695.

Medoued, A., Lebaroud, A., Laifa, A., \& Sayad, D. (2014). Classification of induction machine faults using time frequency representation and particle swarm optimization. Journal of Electrical Engineering and Technology, 9(1), 170-177.

Munshi, A., \& Sampath, W. (2016). CdTe Photovoltaics for Sustainable Electricity Generation. J. Electron. Mater.

Ordaz-Moreno, A., de Jesus Romero-Troncoso, R., Vite-Frias, J. A., Rivera-Gillen, J. R., \& Garcia-Perez, A. (2008). Automatic online diagnosis algorithm for broken-bar detection on induction motors based on discrete wavelet transform for FPGA implementation. IEEE Transactions on Industrial Electronics, 55(5), 2193-2202.

Puche-Panadero, R., Pineda-Sanchez, M., Riera-Guasp, M., Roger-Folch, J., Hurtado-Perez, E., \& Perez-Cruz, J. (2009). Improved resolution of the MCSA method via Hilbert transform, enabling the diagnosis of rotor asymmetries at very low slip. IEEE Transactions on Energy Conversion, 24(1), 52-59.

Rahman, M. M., Baky, M. A. H., \& Islam, A. S. (2017). Electricity from Wind for Off-Grid Applications in Bangladesh: A Techno-Economic Assessment. International Journal of Renewable Energy Development, 6(1), 55.

Sadeghian, A., Ye, Z., \& Wu, B. (2009). Online detection of broken rotor bars in induction motors by wavelet packet decomposition and artificial neural networks. IEEE Transactions on Instrumentation and Measurement, 58(7), 2253-2263.

Sreedharan, S., Ongsakul, W., Singh, J. G., \& Mahapatra, S. S. (2011). Development of PSO-based robust controller for maximising wind penetration. International Journal of Renewable Energy Technology, 3(1), 58-78.

Tavner, P. J. (2008). Review of condition monitoring of rotating electrical machines. IET Electric Power Applications, 2(4), $215-247$

Toke, D. (2015). Renewable Energy Auctions and Tenders: How good are they?. International Journal of Sustainable Energy Planning and Management, 8, 43-56.

Wang, J., Liu, S., Gao, R. X., \& Yan, R. (2012). Current envelope analysis for defect identification and diagnosis in induction motors. Journal of Manufacturing Systems, 31(4), 380-387.

Ye, Z., Wu, B., \& Zargari, N. (2000). Online mechanical fault diagnosis of induction motor by wavelet artificial neural network using stator current. In Industrial Electronics Society, 2000. IECON 2000. 26th Annual Conference of the IEEE (Vol. 2, pp. 1183-1188). IEEE. 